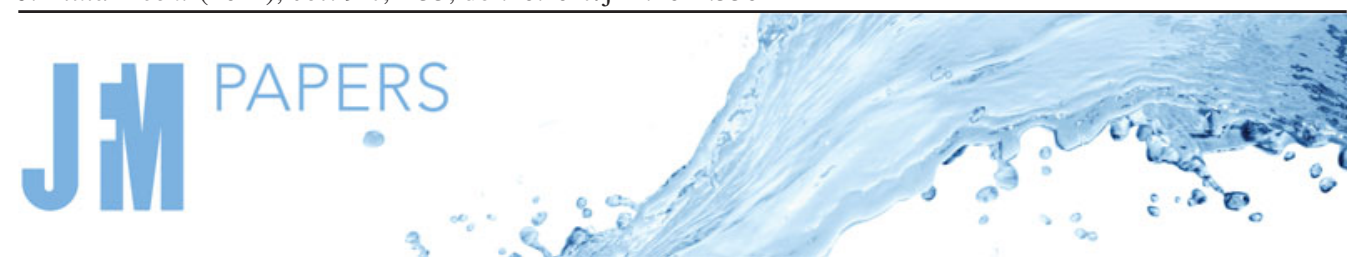

\title{
Self-turbulization in cellularly unstable laminar flames
}

\author{
Zirui Liu ${ }^{1}$, Vishnu R. Unni ${ }^{2}$, Swetaprovo Chaudhuri ${ }^{3}$, Ran Sui ${ }^{1}$, \\ Chung K. Law ${ }^{1}$ and Abhishek Saha ${ }^{2} \dagger$ \\ ${ }^{1}$ Department of Mechanical and Aerospace Engineering, Princeton University, Princeton, NJ 08544, USA \\ ${ }^{2}$ Department of Mechanical and Aerospace Engineering, University of California San Diego, \\ La Jolla, CA 92093, USA \\ ${ }^{3}$ Institute of Aerospace Studies, University of Toronto, Toronto, ON M3H 5T6, Canada
}

(Received 14 October 2020; revised 18 March 2021; accepted 8 April 2021)

It has been suggested that a cellularly unstable laminar flame, which is freely propagating in unbounded space, can accelerate and evolve into a turbulent flame with the neighbouring flow exhibiting the basic characteristics of turbulence. Famously known as self-turbulization, this conceptual transition in the flow regime, which arises from local interactions between the propagating wrinkled flamefront and the flow, is critical in extreme events such as the deflagration-to-detonation transition (DDT) leading to supernova explosions. Recognizing that such a transition in the flow regime has not been conclusively demonstrated through experiments, in this work, we present experimental measurements of flow characteristics of flame-generated 'turbulence' for expanding cellular laminar flames. The energy spectra of such 'turbulence' at different stages of cellular instability are analysed. A subsequent scaling analysis points out that the observed energy spectra are driven by the fractal topology of the cellularly unstable flamefront.

Key words: flames, instability, transition to turbulence

\section{Introduction}

A premixed laminar flame propagating in a quiescent, gravity-free environment can develop intrinsic flamefront cellular instability, which renders the otherwise smooth flamefront wrinkled with a wide range of cell sizes. The instability can be one of two modes, namely, the Darrieus-Landau hydrodynamic instability, caused by the sharp density gradient across the flamefront and promoted at higher pressures owing to reduced

$†$ Email address for correspondence: asaha@eng.ucsd.edu

(C) The Author(s), 2021. Published by Cambridge University Press. This is an Open Access article, distributed under the terms of the Creative Commons Attribution licence (http://creativecommons.org/ licenses/by/4.0/), which permits unrestricted re-use, distribution, and reproduction in any medium, provided the original work is properly cited. 
flame thickness and increased reactivity; and diffusional-thermal instability, which is promoted in mixtures with weaker thermal diffusion compared to mass diffusion and can be identified by a sub-unity Lewis number, $L e<1$, defined as the ratio of thermal to mass diffusivities. Irrespective of the instability mode, once the cellular structure appears on the flamefront, the continuous generation and evolution of new cells will increase the flame surface area and hence, the burning rate, a process known as self-acceleration. Owing to its capability to accelerate without external sources, such as turbulence, these laminar self-accelerating flames have been extensively studied (Gostintsev, Istratov \& Shulenin 1988; Ashurst 1997; Clanet \& Searby 1998; Bradley 1999; Truffaut \& Searby 1999; Wu, Jomaas \& Law 2013; Yang et al. 2016; Beeckmann et al. 2017). Furthermore, it has also been suggested that a self-accelerating laminar flame could attain a 'turbulence-like' behaviour, a phenomenon termed self-turbulization, which in turn could become supersonic through a process termed deflagration-to-detonation transition, or DDT (Sivashinsky 1977; Oran \& Gamezo 2007; Vasil'ev 2012; Clavin \& Searby 2016), which has been analytically and numerically studied (Zeldovich \& Rozlovskii 1947; Khokhlov, Oran \& Wheeler 1997; Akkerman, Law \& Bychkov 2011; Kagan \& Sivashinsky 2017; Kiverin, Yakovenko \& Fortov 2019). The issue of self-turbulization, within this proposed trilogy of events, that is, from self-acceleration, to self-turbulization to DDT, has not been adequately examined and is the subject of the present study.

In the 1970s, Sivashinsky (1977) first suggested the term self-turbulization when he theoretically derived the growth rate of instability in laminar premixed flames subjected to cellular instability, and demonstrated that the propagation speed exceeds that of a stable flame owing to the cellular structure, a behaviour commonly associated with turbulent flames. Later, Bradley (Bradley \& Harper 1994; Bradley 1999) conjectured that the non-uniformity of the flamefront could lead to an inhomogeneity in the adjacent flow due to baroclinic torque leading to a 'turbulent' flow. In attempts to address the existence of self-turbulization, a large number of theoretical (Bradley 1999; Addabbo, Bechtold \& Matalon 2002), computational (Filyand, Sivashinsky \& Frankel 1994; Ashurst 1997) and experimental (Gostintsev et al. 1988; Wu et al. 2013; Yang et al. 2016) investigations have studied the self-similarity nature of flame propagation, and evaluated the acceleration exponent as well as the associated fractal dimension of the flamefront and compared with those of flames in Kolmogorov turbulence. The acceleration exponent, $\alpha$, for cellularly unstable expanding flames is defined through $R_{f} \sim t^{\alpha}$, where $R_{f}$ is the mean flame radius and $t$ the time from ignition. The possible attainment of self-turbulization is assessed by evaluating whether the fractal dimension of the wrinkled two-dimensional (2-D) flamefront reaches the theoretical value of Kolmogorov turbulence, 7/3 (Sreenivasan \& Meneveau 1986; Sreenivasan, Ramshankar \& Meneveau 1989), or equivalently whether $\alpha$ reaches a critical value of 1.5 (Gostintsev et al. 1988). It is then of interest to note that experimental results (Gostintsev et al. 1988; Wu et al. 2013; Yang et al. 2016), obtained by measuring the flame radius $R_{f}$ as function of time, have not shown acceleration exponents exceeding 1.5. Here, we note that the aforementioned tests, through evolution of the flame radius, is an indirect approach, which inherently assumes that self-tubulization should lead to Kolmogorov turbulence-like behaviour, although originally, this was not suggested by Sivashinsky (1977). Indeed, while a direct test is to measure the characteristics of the flow field induced by the wrinkled flamefront and examine if it resembles that of a turbulent flow, experimental measurement of such flow structures has not been systematically attempted, ostensibly due to inherent complications in the measurement. Furthermore, it is also noted that most of the numerical studies (Rastigejev \& Matalon 2006; Yu, Bai \& Bychkov 2015; Creta et al. 2020) have focused on the effects of cellular instability on flame 


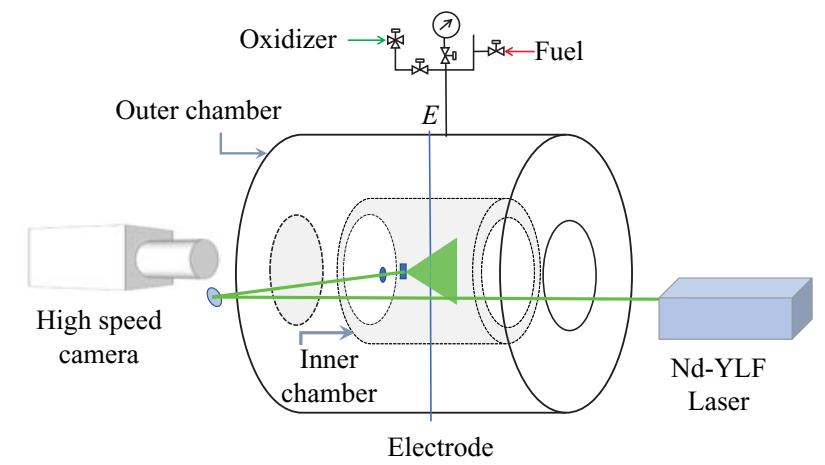

Figure 1. Experimental setup: Dual chamber vessel with high-speed camera, optics and laser used for high-speed particle image velocimetry.

propagation and flame wrinkling, with several (Fogla, Creta \& Matalon 2015; Lamioni et al. 2018) discussing the flow patterns for cellular flames propagating in turbulence. Consequently, they are unrelated to the issues of self-turbulization.

Recognizing the lack of, and hence the need for, understanding the issue of self-turbulization, in this study we present an experimental investigation to provide useful information on the flow induced by cellular flames. Specifically, in the context of expanding cellular flames, we set out to answer the following three questions: (i) What are the primary characteristics of the flow induced by cellularly unstable flames? (ii) Because the flow is induced by the wrinkled flamefront, is there a connection between the cellular flamefront and the flow structure? (iii) Do cellular flames induce a turbulent flow?

\section{Experiments}

We conducted our experiments in a well-vetted, dual-chamber combustion vessel, in which propagation of a centrally ignited, outwardly propagating globally spherical premixed flame could be studied in a constant and high-pressure environment, triggering the Darrieus-Landau instability. The apparatus consists of two cylindrical chambers. The inner chamber of almost unity aspect ratio and filled with the test mixture is situated in an outer chamber of a much larger volume (inner diameter: $114 \mathrm{~mm}$, outer diameter: $167 \mathrm{~mm}$; length: $127 \mathrm{~mm}$ ) and filled with nitrogen $\left(\mathrm{N}_{2}\right)$ having the same pressure as that of the test mixture. The flat ends of the inner chamber are sealed with thick quartz windows for optical access. The connection between the chambers is controlled by four valves (sliding sleeve with multiple holes) which are opened when the test mixture is spark ignited and allowed the expanding flame to come in contact with the inert gas in the outer chamber to quench. Thus, the expansion process mimicks an isobaric process owing to the small volume of the inner chamber compared to the outer chamber. Figure 1 shows the schematic of the apparatus. More details of the chamber can be found in our previous publications (Chaudhuri et al. 2012; Yang et al. 2016; Liu et al. 2020) and in the Supplementary material available at https://doi.org/10.1017/jfm.2021.330. The flame propagation was studied using two diagnostic techniques. High-speed Schlieren imaging was used to capture the flame morphology, the propagation speed and the acceleration exponent, as shown in figures $2(a)$ and $2(b)$. High-speed $(8 \mathrm{kHz}) 2-\mathrm{D}$ particle image velocimetry (PIV) was used to measure the flow dynamics. The mixtures were seeded with a small amount of di-ethyl-hexyl-sebacat droplets $(\sim 1-2 \mu \mathrm{m})$ before ignition. 


\section{Z. Liu and others}

(a)

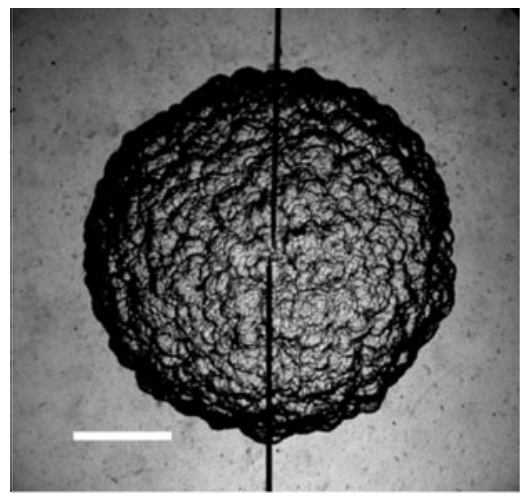

(c)

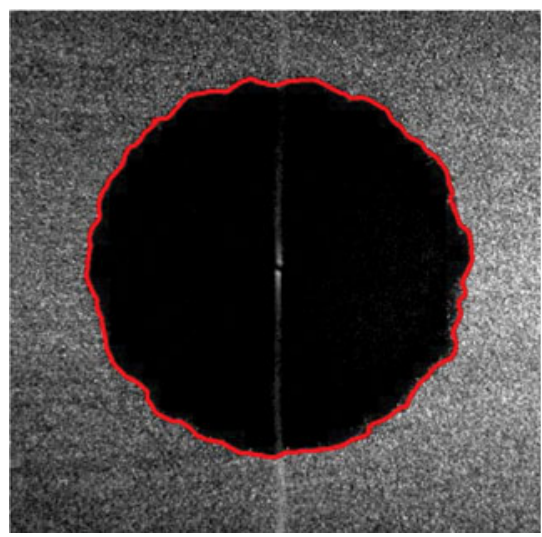

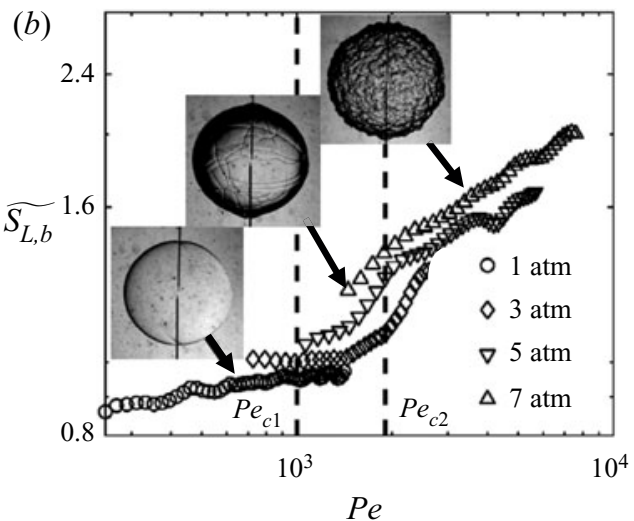

$(d)$

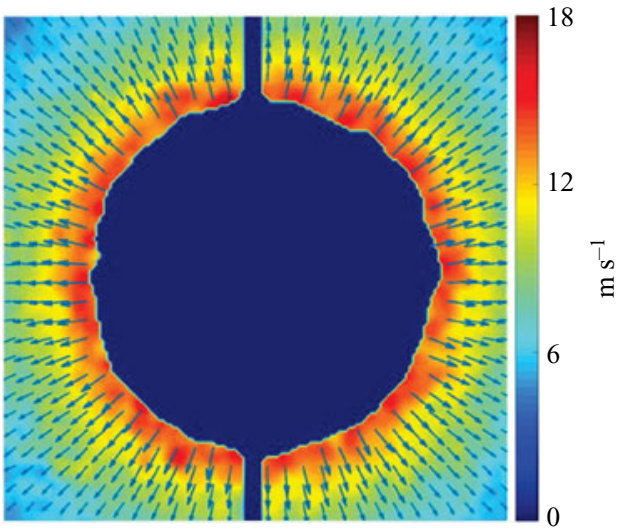

Figure 2. (a) Schlieren image. The inset bar indicates $10 \mathrm{~mm}$. (b) Three-stage behaviour of flame propagation. Here, $P e$, Péclet number; $\widetilde{S_{L, b}}$, flame propagation speed normalized by laminar unstretched flame speed (burned side). Replotted from data presented in Yang et al. (2018). (c) Mie scattering image with edge detection. (d) Vector field from particle image velocimetry. Colour represents magnitude of local velocity.

A planar 2-D laser sheet, which was generated using a high-speed Nd-YLF dual-pulsed laser and associated optics, was placed at the centre of the chamber. During propagation of the flame, the droplets evaporated in the burned side due to high temperature, which resulted in a clear flame boundary in the Mie scattering images captured by a high-speed camera orthogonally placed and synchronized with the laser sheet (shown in figure $2 c$ ). The flamefront in this study, therefore, is essentially the isothermal surface at the droplet boiling point, which does not vary significantly across the prevailing pressure condition. The mean flame radius $R_{f}$ is defined based on the area $\left(A_{f}\right)$ enclosed by the flamefronts in the 2-D Mie scattering images, $R_{f}=\sqrt{A_{f} / \pi}$. The DaVis PIV algorithm and in-house MATLAB codes were used to extract the velocity field around the flamefront (shown in figure $2 d$ ), which was then analysed to yield the specific flow characteristics. The uncertainty of the velocity measurement was evaluated to be less than $4 \%$. In this study, we solely focused on the effect of the Darrieus-Landau hydrodynamic cellular instability, and hence used diffusively neutral $(L e \approx 1)$ stoichiometric (equivalence ratio, $\phi=1$ ) hydrogen $\left(\mathrm{H}_{2}\right)$ /oxygen $\left(\mathrm{O}_{2}\right)$ /nitrogen $\left(\mathrm{N}_{2}\right)$ mixtures at a flame temperature of $2400 \mathrm{~K}$ to minimize stabilization/destabilization effects of diffusional-thermal instability. We also note that the effects of Rayleigh-Taylor instability were rather weak for our experimental 


\begin{tabular}{cccccccc} 
Mixture & $\phi$ & $\frac{Y_{\mathrm{O}_{2}}}{\left(Y_{\mathrm{O}_{2}}+Y_{\mathrm{N}_{2}}\right)}$ & Le & $T_{f}(\mathrm{~K})$ & $\mathrm{P}(\mathrm{atm})$ & $S_{L, 0}\left(\mathrm{~m} \mathrm{~s}^{-1}\right)$ & $\delta_{L, 0}(\mu \mathrm{m})$ \\
$\mathrm{H}_{2} / \mathrm{O}_{2} / \mathrm{N}_{2}$ & 1 & $21 \%$ & \multirow{2}{*}{21} & 2400 & 1 & 2.096 & 20.6 \\
& & & & & 3 & 1.977 & 7.3 \\
& & & & & 5 & 1.823 & 4.8 \\
& & & & & & & \\
& & & & & & &
\end{tabular}

Table 1. Experimental conditions with corresponding flame parameters. The parameters are $\phi$, equivalence ratio; $Y_{i}$, mole fraction of species $i$ in unburned mixture; Le, Lewis number; P, pressure; $S_{L, 0}$, flame speed of laminar planar flame; $\delta_{L, 0}$, thickness of laminar planar flame.

conditions (Bell et al. 2004; Abarzhi et al. 2019) and hence, the reported flame dynamics solely arose from Darrieus-Landau instability.

The fundamental flame properties, such as the laminar unstretched flame speed $\left(S_{L, 0}\right)$ and adiabatic flame temperature $\left(T_{f}\right)$, were calculated numerically using the CHEMKIN one-dimensional flame solver and a reaction model of $\mathrm{H}_{2}$-oxidation proposed by Burke et al. (2012). The experimental conditions and corresponding flame properties are listed in table 1. In this study, we defined the planar flame thickness based on diffusivity, $\delta_{L, 0}=D_{T} / S_{L, 0}$, where $D_{T}$ is the thermal diffusivity of the mixture. The instantaneous propagation speed of the expanding flame, $S_{L, b}=\mathrm{d} R_{f} / \mathrm{d} t$, was obtained from the time derivative of the mean flame radius, $R_{f}$. To study a wide range of $P e$, we experimented with a broad range (1-7 atm) of ambient pressures.

\section{Results}

\subsection{Global propagation}

In our previous study (Yang et al. 2016), it was shown that the propagation of cellularly unstable expanding flames at various pressures can be unified when the normalized flame propagation speed is plotted with the normalized radius, or Péclet number, $P e=R_{f} / \delta_{L, 0}$, somewhat similar to turbulent flames (Chaudhuri et al. 2012). Figure 2(b) shows such a normalized plot for the current experiments, which identifies a three-stage behaviour with two critical $P e^{\prime}$ s. Specifically, the flamefront in the smooth expansion stage, $P e<P e_{c 1} \approx$ 1000 , is free from instabilities (inset of figure $2 b$ ) and hence the propagation is stretch controlled. As the cellular instability is triggered at $P e=P e_{c 1}$, the flame shows sharp acceleration, and over a short $P e$ range, new cells start appearing and spreading over the entire flamefront (inset of figure $2 b)$, which marks a transition stage $\left(P e_{c 1}<P e<P e_{c 2}\right)$. At the saturated stage $\left(P e>P e_{c 2} \approx 1900\right)$, the flamefront becomes fully covered with cells (inset of figure $2 b$ ) and the acceleration attains self-similarity. Yang et al. (2016) showed that for a wide range of conditions, the acceleration exponent $(\alpha)$ lies in the range 1.2-1.4 and the corresponding fractal excess $(d=1-1 / \alpha)$ is within $0.2-0.3$. In a similar, but larger chamber, Huo et al. $(2018,2019)$ also observed the three-stage behaviour with a similar global acceleration exponent. However they noticed an oscillation in the mean propagation speed in the saturated stage. We note that such pulsation occurs for a larger flame and was not observed in our experiments. Furthermore, in this study, we will be analysing the energy spectra from local velocity fluctuation and, as such, oscillation in mean propagation speed or mean velocity is expected to have an insignificant effect on the reported statistics. 


\section{Z. Liu and others}
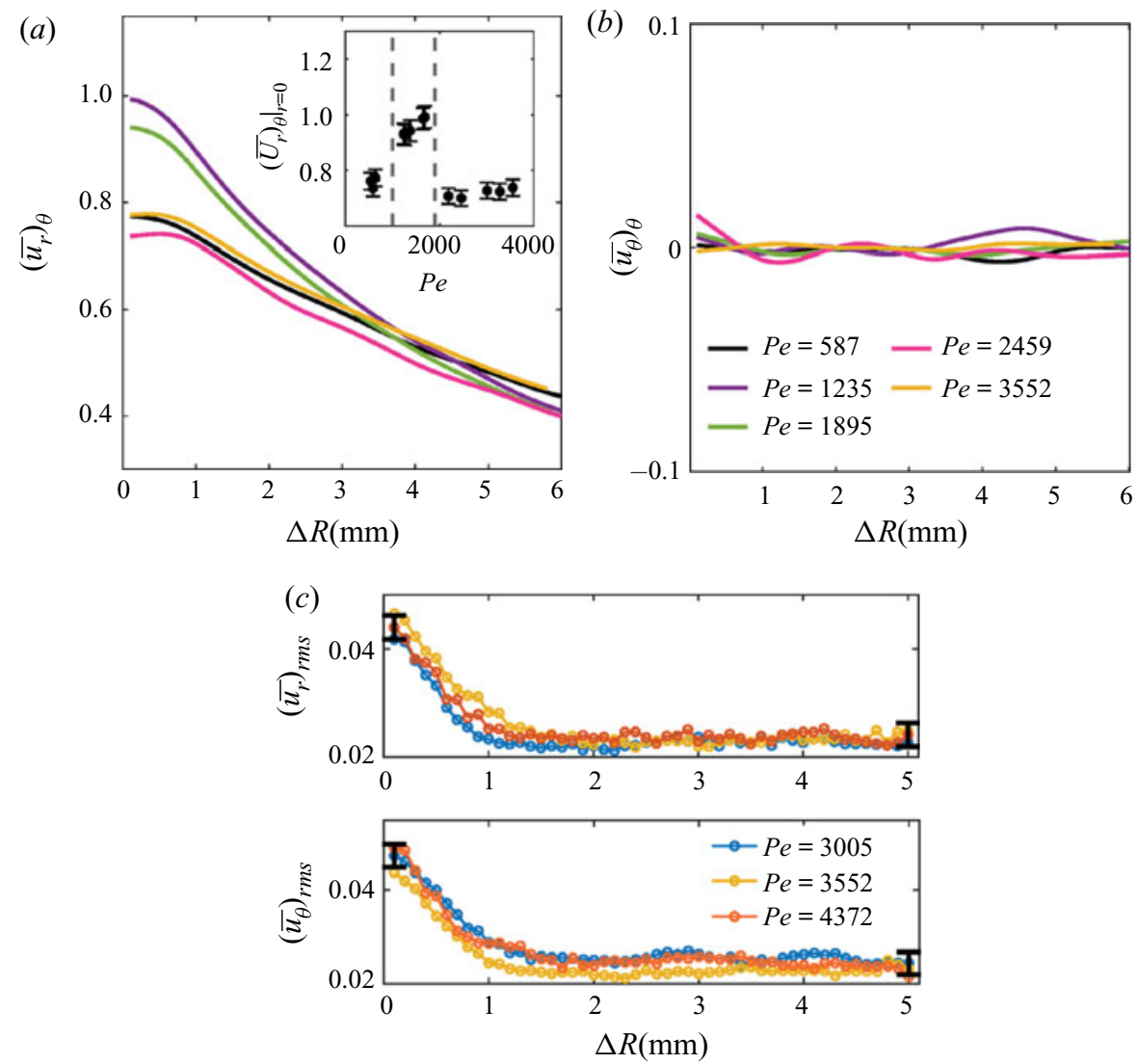

Figure 3. (a) Averaged normalized radial velocity, $\left\langle\overline{U_{r}}\right\rangle_{\theta}$, versus distance away from flamefront, $\Delta R$, at different $P e$. Inset: Averaged normalized radial velocity, $\left\langle\overline{U_{r}}\right\rangle_{\theta}$, on the flamefront $(\Delta R=0)$ versus $P e$. (b) Averaged normalized azimuthal velocity, $\left\langle\overline{U_{\theta}}\right\rangle_{\theta}$, versus distance away from flamefront, $\Delta R$, at different $P e$. (c) Root-mean-square (RMS) of normalized radial and azimuthal velocity fluctuation $\overline{u_{r}}, \overline{u_{\theta}}$ versus distance away from flamefront, $\Delta R$, in the saturated stage. The uncertainties (shown in $\mathrm{c}$ ) for radial and azimuthal velocities are within $\pm 6 \%$.

\subsection{Flow statistics}

Next, we focus our attention on the flow field ahead of the propagating cellular flamefront measured by PIV. To characterize the flow generated by the flame, we first explore the variation of the radial $\left(U_{r}(r, \theta)\right)$ and azimuthal $\left(U_{\theta}(r, \theta)\right)$ components of the velocity in the radial direction. To present the radial variation of these velocities at various flame conditions, we take the azimuthal average of these velocities at any fixed $r$ (averaged quantities denoted as $\langle\cdot\rangle_{\theta}$ ) and normalize the averaged velocity with the flame propagation speed, $S_{L, b}$ (normalized quantities presented as $\overline{(\cdot)}$ ). In figure $3(a)$, we compare the averaged normalized radial velocity, $\left\langle\overline{U_{r}}\right\rangle_{\theta}$, as function of distance from the flamefront, $\Delta R$, for various $P e$. For all $P e,\left\langle\overline{U_{r}}\right\rangle_{\theta}$ is maximum adjacent to the flamefront and decay monotonically with $\Delta R$ as the effect of flame propagation weakens away from the flamefront. Interestingly, the averaged normalized radial velocity at the flamefront $\left(\left.\left\langle\overline{U_{r}}\right\rangle_{\theta}\right|_{\Delta R=0}\right)$ shows a non-monotonic dependence on $P e$, in that at low Pe (smooth expansion stage, $P e<P e_{c 1}$ ) and high $P e$ (saturated stage, $P e>P e_{c 2}$ ), it is smaller compared to that at intermediate $P e$ (transition stage, $P e_{c 1}<P e<P e_{c 2}$ ), as shown in the 

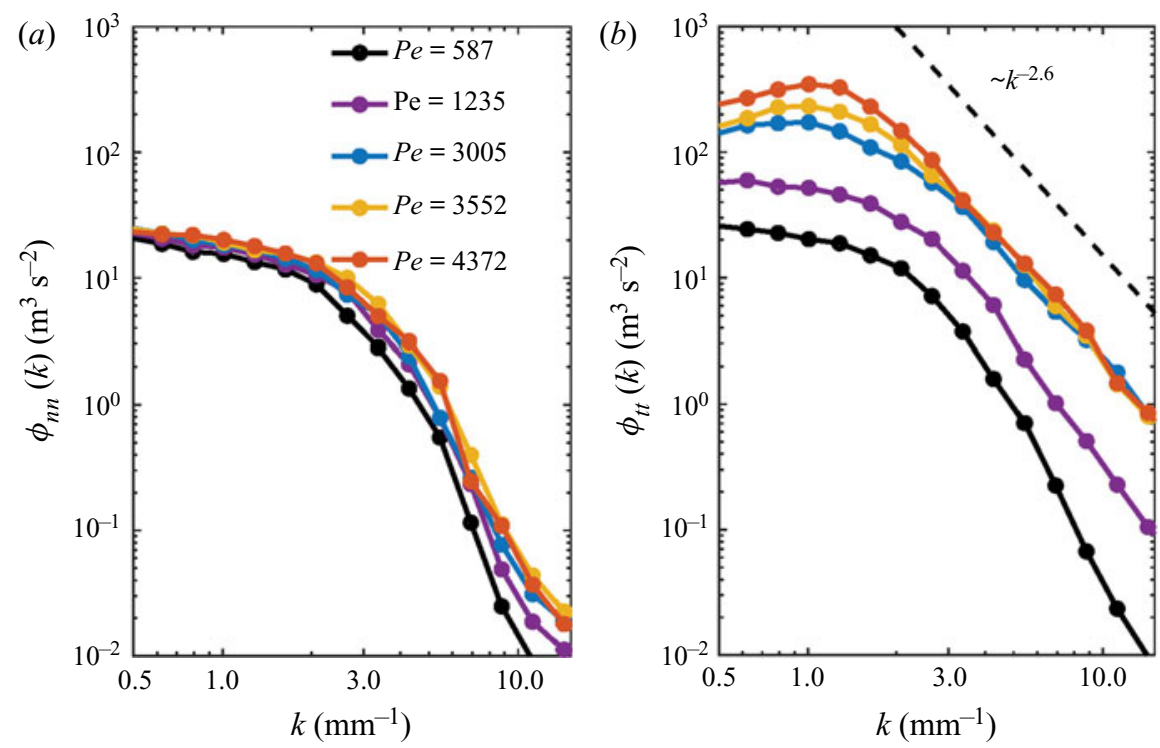

Figure 4. (a) Energy spectra of normal velocity fluctuation $u_{n}$ on the flamefront at different $P e$. (b) Energy spectra of tangential velocity fluctuation $u_{t}$ on the flamefront at different $P e$. Here, $P e=587$, smooth expansion stage; $P e=1235$, transition stage; the rest, saturated stage. The uncertainty of the power spectral density is approximately $\pm 12 \%$.

inset of figure 3(a). At the onset of the transition stage, the flamefront becomes unstable and hence experiences sudden acceleration (figure $2 b$ ), and thereby increasing the radial velocity adjacent to the flamefront significantly before the far field flow could respond. Such dramatic increase, however, is attenuated at the saturated stage, as the cellular structure and flamefront morphology become saturated and the acceleration becomes 'steady', which in turn reduces $\left.\left\langle\overline{U_{r}}\right\rangle_{\theta}\right|_{\Delta R=0}$ to the original value.

The averaged normalized azimuthal velocity, $\left\langle\overline{U_{\theta}}\right\rangle_{\theta}$, however, is much weaker than $\left\langle\overline{U_{r}}\right\rangle_{\theta}$ and does not show any correlated variation along the radial direction, as shown in figure 3(b). The flow structure generated by the flamefront is consumed by the propagating flamefront before it is convected to the far field and hence no particular correlation between near field and far field was observed. This can be further shown through the velocity fluctuations in the radial $\left(u_{r}\right)$ and azimuthal $\left(u_{\theta}\right)$ directions. The RMS of $\overline{u_{r}}$ and $\overline{u_{\theta}}$ monotonically decrease with $\Delta R$, as shown in figure 3(c). Such monotonic decay suggests that the flow far away from the flame is dominated by the mean radial flow generated by thermal expansion and does not possess strong velocity fluctuations.

Recognizing that the flame-generated flow structures were mostly confined in the vicinity of the flamefront, we focus the rest of the analysis on the flow vectors conditioned on the wrinkled flamefront, and analysed them in a flame-coordinate system using the normal and tangential directions of the flamefront. Figure 4 shows the one-dimensional energy spectra $\phi_{n n}, \phi_{t t}$ of the fluctuation velocity in the normal, $u_{n}$, and tangential, $u_{t}$, directions, respectively, measured along the flamefront for five values of $P e$ spanning all three stages of propagation. We observe that $\phi_{n n}(k)$ shows a similar shape at the different stages of the propagation. Furthermore, the energy is large at small wavenumbers (large scales) and rapidly decayed at large wavenumbers (small scales). Although the 
shape of $\phi_{t t}$ is similar to $\phi_{n n}$ at low $P e$, there are some characteristic changes with $P e$ (figure $4 b$ ). First, the spectra shifts to higher energy values as $P e$ increases and the flame moves from the smooth expansion stage $(P e=587)$ to the transition stage $(P e=$ $1235)$ to the saturated stage ( $P e=3005,3552$ and 4372) with the flamefront becoming progressively more wrinkled owing to the appearance of the cellular structure. Moreover, at the saturated stage, the spectra almost overlap $(P e=3005,3552$ and 4372$)$ at various $P e$ and as such, become independent of $P e$. They also attains a power-law dependence, i.e. $\phi_{t t}(k) \sim k^{-\beta}$, where $\beta$ was measured to be approximately 2.6 (the dashed line). The power-law behaviour is observed for length scales, $2 \pi / k$, roughly from the flame radius $\left(R_{f} \sim O(10 \mathrm{~mm})\right)$ to the smallest scales we can resolve in the experiments, which is $O(0.1 \mathrm{~mm}) \sim 20 \delta_{L, 0}$. We note that theoretical analyses (Pelce \& Clavin 1982; Bychkov \& Liberman 2000) have shown that the cut-off limit of Darrieus-Landau instability is approximately $k_{\text {cut-off }} \approx 2 \pi /\left(20 \delta_{L, 0}\right)$, which is close to the experimental resolution. Nevertheless, whether the power-law scaling holds for larger wavenumbers is still to be investigated. The turbulent Reynolds number based on the largest scale $\left(\operatorname{Re}_{t}=u_{r m s} R_{f} / v\right)$ at the saturated stage was in the range of 1500-4000, where $v$ is the kinematic viscosity of unburned gas.

It should be noted that the flow statistics were measured in the meridional plane of the cellularly unstable expanding flame and as such, were 2-D in nature. However, these flames are statistically spherical (Chaudhuri, Wu \& Law 2013; Chaudhuri, Saha \& Law 2015) and as such, orientation of the measurement plane does not affect the statistics.

\subsection{Scaling analysis}

Next, we explore the origin of the power-law dependence observed in the flame-generated flow. Here, we note that in our experiments, the flame propagates in a quiescent environment and as such, the observed energy spectra of the flow adjacent to the flamefront is generated by the wrinkling of the flamefront. Consequently, we present a scaling analysis to connect the energy spectra with the cellular structure. In the Landau limit, every point on the flamefront propagates individually in the normal direction at the speed of $S_{L, 0}$. Defining $S_{w}$ as the projected propagation speed on the mean spherical flamefront for every local infinitesimal flame segment, we can write $S_{w} / S_{L, 0}=|\nabla G|=\sqrt{1+\nabla g \cdot \nabla g}$, where $G$ is the scalar describing the flamefront in the well-known G-equation (Peters 1999). We can also define $G=R_{f}+g-r$, where $g$ is the fluctuation of the flamefront from the mean surface for a mildly wrinkled flamefront, and $r$ is the radial distance from the centre of the flame. Such a definition ensures $G>0$ in the unburned side, $G<0$ in the burned side and $G=0$ on the flamefront, a convention commonly followed in the G-equation representation. Because the flow is induced by the flame, the kinetic energy of the flow (E) must arise from flame propagation, or $E \sim S_{w}^{2}$. Considering the contribution from the flow velocity in the normal $U_{n}$ and tangential $U_{t}$ components, we can write

$$
U_{n}^{2}+U_{t}^{2} \sim S_{L, 0}^{2}(1+\nabla g \cdot \nabla g) .
$$

In the Landau limit, for adjacent fluid particles, $U_{n}^{2}$ scales as $S_{L, 0}^{2}$, which, along with (3.1), suggests that $U_{t}^{2} \sim S_{L, 0}^{2} \nabla g \cdot \nabla g$. We notice that $U_{n}^{2}$ is independent of $g$ or the geometry of the wrinkled flamefront at all stages of propagation or Pe (figure $4 a$ ). Consequently, fluctuation of the normal velocity $u_{n}$ is mainly determined by the disturbance on the large scales in the system, while decaying rapidly on the small scales. This explains the similar energy spectra at all values of $P e$, as the disturbance on the large scale is independent 
(a)
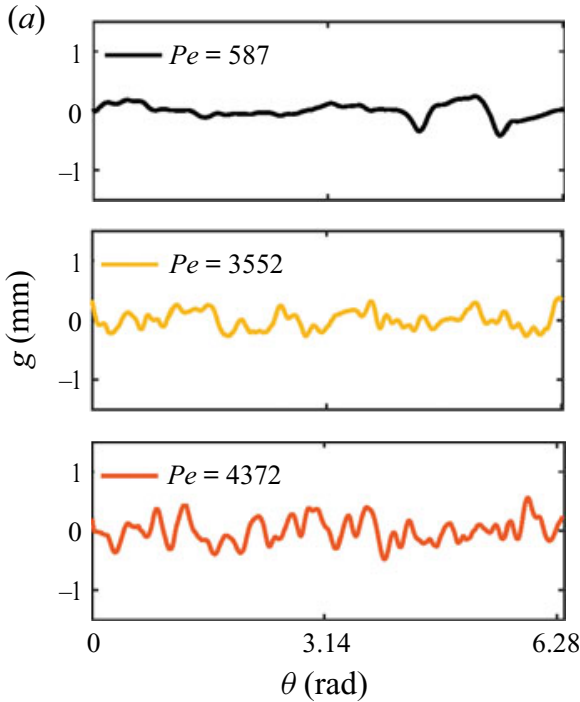

(b)

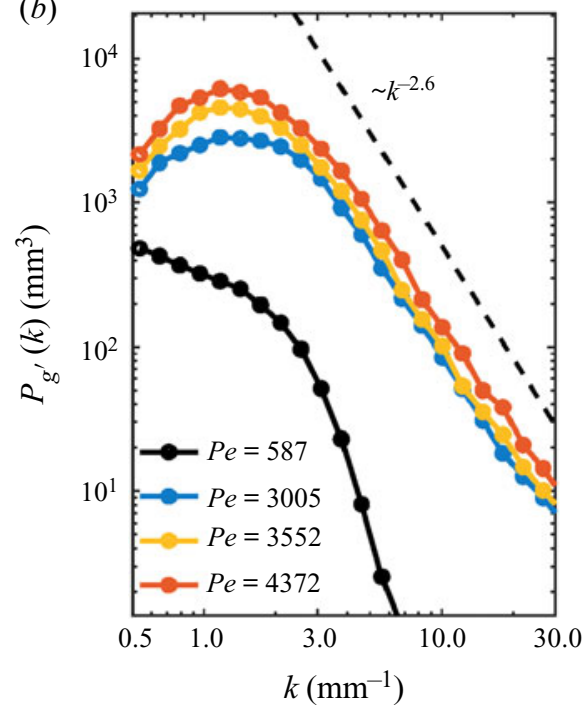

Figure 5. (a) Flamefront fluctuation $g$ versus $\theta$ in the smooth expansion stage and the saturated stage. In the smooth expansion stage, only large cracks are present on the flamefront, while in the saturated stage, small cellular structures are generated on the flamefront. (b) Power spectra density of $\mathrm{d} g / \mathrm{d} x$ of the flamefront. Here wavenumber $k$ is based on the distance along the flamefront. The smallest resolved scale is controlled by the resolution of the Mie scattering images. The uncertainty for edge detection is approximately $\pm 1 \%$, and for $P S D$ is $\pm 2 \%$.

of the development of the cellular instability. For fluctuation of the tangential velocity $u_{t}$ at low $P e$ when the flame is free from instability, the energy spectra is the same as that from disturbance. However, as $P e$ increases, cellular instability induces strong tangential velocity structures and the influence of disturbance is suppressed.

Now, we closely explore the relation between $U_{t}^{2}$ and the flame geometry or $g$. First we note that the scaling $U_{t}^{2} \sim \nabla g \cdot \nabla g$ suggests that the power spectral density (PSD) of $U_{t}$ should scale with the $P S D$ of $|\nabla g|$, denoted as $P_{g^{\prime}}$. Since $P S D$ of the tangential velocity $U_{t}$ and its fluctuation $u_{t}=U_{t}-\left\langle U_{t}\right\rangle$ is equivalent for $k>0$, we have $\phi_{t t}(k) \sim P_{g^{\prime}}(k)$. From the experiments, we measured $g$ (shown in figure $5 a$ ) and evaluated $|\nabla g|$ and $P_{g^{\prime}}(k)$, as shown in figure 5(b). At low Pe (smooth expansion stage), the flame edge $(g)$ possesses less oscillation while at large $\mathrm{Pe}$ (saturated stage), it becomes highly oscillating (figure $5 a$ ). It is then interesting to note that $P_{g^{\prime}}$ (figure $5 b$ ) also shows a power-law dependence with wavenumber in the form of $k^{-2.6}$, which is very similar to $\phi_{t t}$, hence confirming our scaling.

Next, we attempt another scaling analysis to assess the theoretical approximation of $\beta$ in the scaling of $\phi_{t t}(k) \sim P_{g^{\prime}}(k) \sim k^{-\beta}$. Studies (Liberman et al. 2004; Yu et al. 2015; Yang et al. 2016) have confirmed that cellularly unstable laminar flamefronts are fractal in nature. The perimeter, $L$, of the 2-D cross-section of such a wrinkled fractal expanding flame scales with the mean radius $R_{f}$, as $L \sim R_{f}^{D}$, where $D$ is the fractal dimension of the flame edge. Here, $L$ can be obtained by integrating the arc length of the flamefront $R_{f}+g(\theta)$ in the polar coordinate (Strang 1991),

$$
L=\int_{0}^{2 \pi} \sqrt{\left(R_{f}+g\right)^{2}+\left(\frac{\mathrm{d}\left(R_{f}+g\right)}{\mathrm{d} \theta}\right)^{2}} \mathrm{~d} \theta .
$$




\section{Z. Liu and others}

Because the cellularly unstable flamefronts are mildly wrinkled, i.e. $R_{f} \gg g$, and $R_{f}$ is independent of $\theta$, we find

$$
L \approx \int_{0}^{2 \pi} \sqrt{R_{f}^{2}+\left(\frac{\mathrm{d} g}{\mathrm{~d} \theta}\right)^{2}} \mathrm{~d} \theta=R_{f} \int_{0}^{2 \pi} \sqrt{1+\frac{1}{R_{f}^{2}}\left(\frac{\mathrm{d} g}{\mathrm{~d} \theta}\right)^{2}} \mathrm{~d} \theta .
$$

Using $L \sim R_{f}^{D}$, (3.3) can be reduced to

$$
\int_{0}^{2 \pi} \sqrt{1+\left(\frac{\mathrm{d} g}{R_{f} \mathrm{~d} \theta}\right)^{2}} \mathrm{~d} \theta \sim R_{f}^{D-1}
$$

One can show that $\left(1 / R_{f}\right)(\mathrm{d} g / \mathrm{d} \theta) \sim R_{f}^{D-1}$, as it is the only term on the left-hand side of (3.4), which is a function of $R_{f}$. As a result, we have

$$
|\nabla g| \approx\left|\frac{1}{R_{f}} \frac{\mathrm{d} g}{\mathrm{~d} \theta}\right| \sim R_{f}^{D-1}
$$

The cellular structure on the flamefront is bounded by two length scales, the flame thickness scale $\left(\delta_{L, 0}\right)$ and the flame radius scale $\left(R_{f}\right)$ (Matalon 2007), and as such, the spectrum of $g$, which describes the flamefront geometry, can be expressed as

$$
g(\theta) \sim \int_{2 \pi / R_{f}}^{2 \pi / \delta_{L, 0}} A(k) \sin \left(k R_{f} \theta+\psi_{k}\right) \mathrm{d} k,
$$

where $k$ represents the wavenumber along the flamefront and $\psi_{k}$ is the phase angle. Assuming $A(k)$, the amplitude at wavenumber $k$ follows a power law $A(k) \sim k^{-\gamma}$. By differentiating it with respect to $\theta$ and substituting $A(k) \sim k^{-\gamma}$, we obtain

$$
\frac{\mathrm{d} g}{\mathrm{~d} \theta} \sim R_{f} \int_{2 \pi / R_{f}}^{2 \pi / \delta_{L, 0}} k^{1-\gamma} \cos \left(k R_{f} \theta+\psi_{k}\right) \mathrm{d} k
$$

The amplitude of $\mathrm{d} g / \mathrm{d} \theta$ at wavenumber $k$ scales with $k^{1-\gamma}$, thus, PSD of $\mathrm{d} g / \mathrm{d} \theta$ has a $k^{2(1-\gamma)}$ dependence; in other words, $\beta=-2(1-\gamma)$. Now rearranging (3.7) and substituting $\lambda=k R_{f}$ as a normalized wavenumber, we have

$$
\frac{1}{R_{f}} \frac{\mathrm{d} g}{\mathrm{~d} \theta} \sim R_{f}^{\gamma-2} \int_{2 \pi}^{2 \pi P e} \lambda^{1-\gamma} \cos \left(\lambda \theta+\psi_{\lambda}\right) \mathrm{d} \lambda .
$$

It can be shown (see Supplementary material) that the integral in (3.8) converges to a constant value for any $\theta$ and $\psi$ for $\gamma>1$ at large $P e$, and hence is independent of $R_{f}$. This leads to $\left|d g /\left(R_{f} d \theta\right)\right| \sim R_{f}^{\gamma-2}$, and by comparing with (3.5), we find $\gamma=D+1$ or $\beta=2 D$. Using the experimentally measured fractal dimension $(D=1+d \approx 1.25 \pm 0.05)$ in the saturated stage for our flames (Yang et al. 2016), we expect $\beta=2.5 \pm 0.1$, which is close to the observed value of 2.6 shown in figures $4(b)$ and $5(b)$.

\section{Concluding remarks}

We conclude by revisiting the questions posed at the beginning. In terms of flow characteristics, we have shown that for an expanding cellularly (Darrieus-Landau) unstable flame, the far field flow is dominated by a radial mean flow, owing to thermal expansion, and the RMS of the velocity is rather weak. Close to the flame, however, 


\section{Self-turbulization in cellularly unstable laminar flames}

the flow shows turbulence-like behaviour. In particular, the energy spectra from the tangential velocity component approach a power-law behaviour in the saturated stage of the propagation. The spectra from the normal component of velocity, however, remains unaltered between the stable and unstable flames. Further analysis based on the G-equation shows that the spectra from the tangential velocity arise from the wrinkling of the cellular flamefronts. We identify that the observed power-law dependence in the energy spectra in the flow is directly related to the fractal dimension of the wrinkled flamefront. We end this exposition with the understanding that the flow adjacent to a cellularly unstable flamefront does become 'turbulent'. Such turbulence, generated by the propagating wrinkled flamefront, has a strong directionality and hence, is anisotropic.

Supplementary material. Supplementary material is available at https://doi.org/10.1017/jfm.2021.330.

Acknowledgements. The authors sincerely thank Prof C. Sun, Prof M. Mueller, Prof L. Deike and Dr K. Kokmanian for helpful discussions.

Funding. This work was supported by the US National Science Foundation (CBET, grant no. 1827287).

Declaration of interests. The authors report no conflict of interest.

\section{Author ORCIDs.}

(c) Vishnu R. Unni https://orcid.org/0000-0002-8830-9779;

D Swetaprovo Chaudhuri https://orcid.org/0000-0003-4109-8633;

(ㄷ) Ran Sui https://orcid.org/0000-0003-3758-7506;

() Abhishek Saha https://orcid.org/0000-0001-9619-6623.

\section{REFERENCES}

Abarzhi, S.I., Ilyin, D.V., Goddard, W.A. \& Anisimov, S.I. 2019 Interface dynamics: mechanisms of stabilization and destabilization and structure of flow fields. Proc. Natl Acad. Sci. USA 116 (37), $18218-18226$.

Addabio, R., Bechtold, J.K. \& Matalon, M. 2002 Wrinkling of spherically expanding flames. Proc. Combust. Inst. 29 (2), 1527-1535.

AKkerman, V., LaW, C.K. \& BychKov, V. 2011 Self-similar accelerative propagation of expanding wrinkled flames and explosion triggering. Phys. Rev. E 83, 026305.

ASHURST, W.T. 1997 Darrieus-Landau instability, growing cycloids and expanding flame acceleration. Combust. Theor. Model. 1 (4), 405-428.

Beeckmann, J., Hesse, R., Kruse, S., Berens, A., Peters, N., Pitsch, H. \& Matalon, M. 2017 Propagation speed and stability of spherically expanding hydrogen/air flames: experimental study and asymptotics. Proc. Combust. Inst. 36 (1), 1531-1538.

Bell, J.B., Day, M.S., Rendleman, C.A., Woosley, S.E. \& Zingale, M. 2004 Direct numerical simulations of type ia supernovae flames. II. The Rayleigh-Taylor instability. Astrophys. J. 608 (2), 883-906.

BRADLEY, D. 1999 Instabilities and flame speeds in large-scale premixed gaseous explosions. Phil. Trans. R. Soc. A 357 (1764), 3567-3581.

BRAdLEY, D. \& HARPER, C.M. 1994 The development of instabilities in laminar explosion flames. Combust. Flame 99 (3-4), 562-572.

Burke, M.P., Chaos, M., Ju, Y., Dryer, F.L. \& Klippenstein, S.J. 2012 Comprehensive $\mathrm{H}_{2} / \mathrm{O}_{2}$ kinetic model for high-pressure combustion. Intl J. Chem. Kinet. 44 (7), 444-474.

Bychiov, V.V. \& Liberman, M.A. 2000 Dynamics and stability of premixed flames. Phys. Rep. 325 (4-5), $115-237$.

Chaudhuri, S., Saha, A. \& Law, C.K. 2015 On flame-turbulence interaction in constant-pressure expanding flames. Proc. Combust. Inst. 35 (2), 1331-1339.

Chaudhuri, S., Wu, F. \& LaW, C.K. 2013 Scaling of turbulent flame speed for expanding flames with Markstein diffusion considerations. Phys. Rev. E 88 (3), 033005.

Chaudhuri, S., Wu, F., ZHu, D. \& LAW, C.K. 2012 Flame speed and self-similar propagation of expanding turbulent premixed flames. Phys. Rev. Lett. 108, 044503. 


\section{Z. Liu and others}

Clanet, C. \& Searby, G. 1998 First experimental study of the Darrieus-Landau instability. Phys. Rev. Lett. 80 (17), 3867.

Clavin, P. \& Searby, G. 2016 Combustion Waves and Fronts in Flows. Cambridge University Press.

Creta, F., Lapenna, P.E., Lamioni, R., Fogla, N. \& Matalon, M. 2020 Propagation of premixed flames in the presence of Darrieus-Landau and thermal diffusive instabilities. Combust. Flame 216, 256-270.

Filyand, L., Sivashinsky, G.I. \& Frankel, M.L. 1994 On self-acceleration of outward propagating wrinkled flames. Physica D 72 (1-2), 110-118.

Fogla, N., Creta, F. \& Matalon, M. 2015 Effect of folds and pockets on the topology and propagation of premixed turbulent flames. Combust. Flame 162 (7), 2758-2777.

Gostintsev, Y.A., Istratov, A.G. \& Shulenin, Y.V. 1988 Self-similar propagation of a free turbulent flame in mixed gas mixtures. Combust. Explos. Shock Waves 24 (5), 563-569.

Huo, J., SAhA, A., REN, Z. \& LAw, C.K. 2018 Self-acceleration and global pulsation in hydrodynamically unstable expanding laminar flames. Combust. Flame 194, 419-425.

Huo, J., SAha, A., Shu, T., Ren, Z. \& LAw, C.K. 2019 Self-acceleration and global pulsation in expanding laminar $\mathrm{H}_{2}-\mathrm{O}_{2}-\mathrm{N}_{2}$ flames. Phys. Rev. Fluids 4 (4), 043201.

KagAn, L. \& Sivashinsky, G. 2017 Transition to detonation of an expanding spherical flame. Combust. Flame 175, 307-311.

Khokhlov, A.M., Oran, E.S. \& WheEler, J.C. 1997 A theory of deflagration-to-detonation transition in unconfined flames. Combust. Flame 108 (4), 503-517.

Kiverin, A.D., Yakovenko, I.S. \& Fortov, V.E. 2019 Mechanism of detonation formation upon free flame propagation in an unconfined space. Dokl. Phys. 64, 449-452.

Lamioni, R., LApenna, P.E., Troiani, G. \& CRetA, F. 2018 Flame induced flow features in the presence of Darrieus-Landau instability. Flow Turbul. Combust. 101 (4), 1137-1155.

Liberman, M.A., Ivanov, M.F., Peil, O.E., Valiev, D.M. \& Eriksson, L.-E. 2004 Self-acceleration and fractal structure of outward freely propagating flames. Phys. Fluids 16 (7), 2476-2482.

LiU, Z., Unni, V.R., Chaudhuri, S., LAW, C.K. \& SAHA, A. 2020 Local statistics of laminar expanding flames subjected to Darrieus-Landau instability. Proc. Combust. Inst. 38 (2), 1993-2000.

Matalon, M. 2007 Intrinsic flame instabilities in premixed and nonpremixed combustion. Annu. Rev. Fluid Mech. 39, 163-191.

ORAN, E.S. \& GAMEZO, V.N. 2007 Origins of the deflagration-to-detonation transition in gas-phase combustion. Combust. Flame 148 (1-2), 4-47.

Pelce, P. \& Clavin, P. 1982 Influence of hydrodynamics and diffusion upon the stability limits of laminar premixed flames. J. Fluid Mech. 124, 219-237.

Peters, N. 1999 The turbulent burning velocity for large-scale and small-scale turbulence. J. Fluid Mech. 384, 107-132.

RAstigeJeV, Y. \& MATAlon, M. 2006 Nonlinear evolution of hydrodynamically unstable premixed flames. J. Fluid Mech. 554, 371-392.

SIVASHINSKY, G.I. 1977 Nonlinear analysis of hydrodynamic instability in laminar flames-I. Derivation of basic equations. Acta Astronaut. 4 (11), 1177-1206.

SREenivasAn, K.R. \& MeneveAu, C. 1986 The fractal facets of turbulence. J. Fluid Mech. 173, $357-386$.

Sreenivasan, K.R., Ramshankar, R. \& Meneveau, C. 1989 Mixing, entrainment and fractal dimensions of surfaces in turbulent flows. Proc. R. Soc. Lond. A 421 (1860), 79-108.

Strang, G. 1991 Calculus. Wellesley-Cambridge Press.

Truffaut, J.-M. \& SEARBy, G. 1999 Experimental study of the Darrieus-Landau instability on an inverted-'V' flame, and measurement of the Markstein number. Combust. Sci. Technol. 149 (1-6), $35-52$.

VASIL'EV, A.A. 2012 Optimization of the deflagration-to-detonation transition. Combust. Explos. Shock Waves 48 (3), 269-277.

Wu, F., JOMAAS, G. \& LAW, C.K. 2013 An experimental investigation on self-acceleration of cellular spherical flames. Proc. Combust. Inst. 34 (1), 937-945.

YAnG, S., SAHA, A., LiU, Z. \& LAW, C.K. 2018 Role of Darrieus-Landau instability in propagation of expanding turbulent flames. J. Fluid. Mech. 850, 784-802.

YAnG, S., SAHA, A., Wu, F. \& LAW, C.K. 2016 Morphology and self-acceleration of expanding laminar flames with flame-front cellular instabilities. Combust. Flame 171, 112-118.

YU, R., BAI, X.-S. \& BYChKOV, V. 2015 Fractal flame structure due to the hydrodynamic Darrieus-Landau instability. Phys. Rev. E 92 (6), 063028.

ZEldovich, Y.B. \& RozlovsKiI, A.I. 1947 On the onset of unstable normal burning: transition of a spherical flame to detonation. Dokl. Akad. Nauk SSSR 57, 365-368. 